$1-1-2012$

\title{
Detection of evolving injury to the brachial plexus during transaxillary robotic thyroidectomy.
}

Adam Luginbuhl

Thomas Jefferson University

Daniel M Schwartz

Surgical Monitoring Associates, Inc.

Anthony K Sestokas

Surgical Monitoring Associates, Inc.

David Cognetti

Thomas Jefferson University

Eollow this and additional works at: https://jdc.jefferson.edu/otofp Edmund Pribitkin

JPlatis ofeffersem atringegosityy Commons

Let us know how access to this document benefits you

\section{Recommended Citation}

Luginbuhl, Adam; Schwartz, Daniel M; Sestokas, Anthony K; Cognetti, David; and Pribitkin, Edmund, "Detection of evolving injury to the brachial plexus during transaxillary robotic thyroidectomy." (2012). Department of Otolaryngology - Head and Neck Surgery Faculty Papers. Paper 15.

https://jdc.jefferson.edu/otofp/15

This Article is brought to you for free and open access by the Jefferson Digital Commons. The Jefferson Digital Commons is a service of Thomas Jefferson University's Center for Teaching and Learning (CTL). The Commons is a showcase for Jefferson books and journals, peer-reviewed scholarly publications, unique historical collections from the University archives, and teaching tools. The Jefferson Digital Commons allows researchers and interested readers anywhere in the world to learn about and keep up to date with Jefferson scholarship. This article has been accepted for inclusion in Department of Otolaryngology - Head and Neck Surgery Faculty Papers by an authorized administrator of the Jefferson Digital Commons. For more information, please contact: JeffersonDigitalCommons@jefferson.edu. 


\section{As submitted to:}

\section{Laryngoscope}

\section{And later published as:}

\section{Detection of Evolving Injury to the Brachial Plexus During Transaxillary Robotic Thyroidectomy \\ Volume 122, Issue 1, January 2012, Pages 110-115 DOI: 10.1002/lary.22429}

Adam Luginbuhl MD ${ }^{1}$, Daniel M. Schwartz, Ph.D., ${ }^{2}$, Anthony K. Sestokas, Ph.D. ${ }^{2}$, David P. Maguire, $M^{3}$, David Cognetti MD ${ }^{1}$, Edmund Pribitkin $M D^{1}$

${ }^{1}$ Department of Otolaryngology Head and Neck Surgery, Thomas Jefferson University, ${ }^{2}$ Surgical Monitoring Associates, Inc., ${ }^{3}$ Department of Anesthesiology, Thomas Jefferson University

Running Title: Neuromonitoring in robotic thyroidectomy

Corresponding Author:

Edmund Pribitkin, MD

Dept of Otolaryngology Head and Neck Surgery

Thomas Jefferson University

925 Chestnut St, $6^{\text {th }}$ floor

Philadelphia, PA 19107

Edmund.pribitkin@jefferson.edu

Phone: 215-955-6784

Fax: 215-923-6784

Disclosures: None of the authors have disclosures to make.

Conflict of Interest: None 
Presented at Combined Triologic Society Meeting in Scottsdale AZ, January $27-30^{\text {th }}$ 2011. 


\begin{abstract}
Introduction: Continuous intraoperative neuromonitoring (IONM) of transcranial electric motor (tceMEP) and somatosensory (SSEP) evoked potentials has gained universal acceptance as an efficacious method for detecting emerging positional brachial plexopathy or peripheral nerve compression during spinal and shoulder surgery. This has implications for transaxillary thyroid surgery.

Methods Study Design: Case report with literature review. The patient underwent robotic transaxillary thyroid surgery with continuous tceMEP and SSEP monitoring of brachial plexus function. We present detailed IONM data depicting emergence of positional brachial plexopathy.

Results: Significant amplitude loss of both IONM modalities identified evolving positional plexopathy, which resolved upon upper extremity repositioning and conversion to an open procedure. No permanent nerve injury or deficit was noted following surgery.

Conclusions: Given the potential for brachial plexus injury during robotic transaxillary thyroid surgery secondary to arm positioning, we recommend that continuous tceMEP and SSEP monitoring be considered during such procedures.

Level of Evidence: IV
\end{abstract}




\section{Introduction}

Injury to the brachial plexus is among the most frequent complications of surgery and often the primary reason for anesthesia-related malpractice claims $s^{i, i i}$. While such positional-based nerve injuries have been noted in both spine and cardiothoracic surgery, there are no such reports in head and neck surgery. iii,iv,v,vi

The recent introduction of transaxillary robotic thyroidectomy, however, presents a unique patient positioning challenge in that the upper extremity is placed in 180 degrees of abduction. For example, in a series of 338 patients who underwent transaxillary robotic thyroidectomy, Kang et al. reported one $(0.3 \%)$ case of postoperative ipsilateral arm paresis. ${ }^{\text {vii }}$ While a single case of unidentified brachial plexopathy may not give rise to significant surgical concern, the medical-legal implications as evidenced by the American Society of Anesthesiologists practice advisory for the prevention of perioperative peripheral neuropathies should not be underestimated. $^{1-2}$

The brachial plexus is responsible for the motor innervations to the muscles of the upper limb, with the exception of the trapezius and levator scapula. The plexus passes in contact with the clavicle anteriorly, first rib inferiorly and the humeral head posterio-laterally and this proximity to these movable bony structures predisposes the neural elements to stretch or compression injury when the arm and shoulder are placed in extreme abduction. Given its widespread use and success in spine surgery, intraoperative neuromonitoring (IONM) should help identify emerging brachial 
plexopathy and assist in determining the need to convert the transaxillary approach to a conventional open thyroidectomy.

We present the first known case report detailing multimodality intraoperative neurophysiologic monitoring detection of evolving positional brachial plexus injury during transaxillary robotic thyroidectomy.

\section{Case Report:}

A 54-year-old morbidly obese, diabetic female presented with a cold left thyroid nodule on nuclear scan post radioactive iodine treatment for hyperthyroidism. FNA demonstrated follicular cells with papillary hyperplasia. Following lengthy discussions regarding the advantages and disadvantages of conventional open versus robotassisted transaxillary thyroidectomy, she consented to the latter, based on factors related to cosmesis.

\section{Intraoperative Neuromonitoring Methods:}

Bilateral multimodality intraoperative neurophysiologic monitoring was used to provide continuous surveillance of neural structures at-risk for iatrogenic injury secondary to surgical or positioning insult including the recurrent laryngeal nerve (RLN) and brachial plexus. A commercially available portable neuromonitoring system (Axon Elite, Axon Systems, Hauppauge, New York) was used for stimulation and recording of all 
monitoring modalities. RLN monitoring was performed with both spontaneous (spEMG) and stimulated (stEMG) electromyography (EMG) using a NIM EMG endotrachial tube (Medtronics, Jacksonville, FL). Upper extremity multi-myotomal transcranial electric motor (tceMEPs) and ulnar nerve cortical somatosensory evoked potentials (UNSSEPs) were elicited according to principals of practice described by Schwartz, et al. ${ }^{4}$ tceMEPs were recorded from the deltoid $(\mathrm{DL})$, biceps $(\mathrm{BI})$, extensor carpi radialis $(\mathrm{EC})$, triceps (TR) and first dorsal interosseous (FD) muscles following electrical stimulation with a brief, high voltage (400-1000 V) anodal pulse train (pulse width $=50 \mathrm{uS}, \mathrm{N}$ pulses $=3-7$, inter-pulse interval $=1-5)$. The multi-pulse stimulus was delivered between two subdermal needle electrodes (Rhythmlink, Columbia, SC) inserted subcutaneously over motor cortex regions C1 and C2 (International 10-20 System).

Sequential left-right ulnar nerve SSEPs were elicited to square-wave electrical pulses (pulse width $=300 \mu \mathrm{S}$ ) delivered to the ulnar nerve at a rate of $4.7 / \mathrm{sec}$. with stimulation intensity set to achieve maximum SSEP amplitude. Cortical SSEP recordings were obtained from subdermal needle electrodes (Rhythmlink, Columbia, SC) inserted at $\mathrm{Cp} 3$ and $\mathrm{Cp} 4$, and referenced to Fpz (International 10-20 System).

A tailored anesthesia protocol was used to optimize multimodality intraoperative neurophysiologic monitoring response amplitudes. Anesthesia was induced with propofol (200 mg) and maintained with total intravenous delivery of propofol (150-175 $\mathrm{mg} / \mathrm{kg} / \mathrm{min}$ ), remifentanil (re: weight), and intermittent low-dose (1-2 mg) midazolam. Oxygen and air were the only inhaled gases. Central temperature was maintained between $35^{\circ} \mathrm{C}$ and $36^{\circ} \mathrm{C}^{3,5}$. 
Initial baseline spEMG, tceMEPs and UNSSEPs were recorded prior to positioning and following clearance of succinylcholine from the neuromuscular junction, as demonstrated by full recovery of response amplitude from first dorsal interosseous muscle to train-of-four (TOF) electrical stimulation of the left ulnar nerve. Thereafter, no neuromuscular blockade was used for the remainder of the case so as not to compromise either EMG or tceMEP signal amplitudes. All neuromonitoring was performed and interpreted by two board-certified Ph.D. surgical neurophysiologists.

\section{Surgical and Neuromonitoring Results}

The patient was positioned with the neck slightly extended. Her left arm was rotated to full abduction at 180 degrees and supported on an armrest wrapped in protective gel pads as illustrated in Figure 1. Incisions were made and dissection proceeded in a traditional manner. Adequate visualization of the thyroid was obtained, the da Vinci Robot (Intuitive Surgical, Sunnyvale CA) was docked, recurrent laryngeal nerve identified and the superior pole was divided.

Post-intubation, pre-positioning baseline transcranial electric motor evoked potentials were recorded successfully from left deltoid, biceps, extensor carpi radialis, triceps and first dorsal interosseous muscles (Figure 2). tceMEPs recorded from the right deltoid, extensor carpi radialis and first dorsal interosseous muscles served as contralateral controls. Baseline somatosensory evoked potentials to stimulation of the left and right ulnar nerves were bilaterally symmetrical for latency and amplitude (Figure 
2). There were no remarkable changes either in somatosensory or transcranial electric motor evoked potential amplitudes following left arm abduction to 180 degrees.

During the early stages of thyroid dissection, the transcranial electric motor evoked potentials from the left upper extremity myotomes (i.e. operative side) showed remarkable amplitude attenuation across all myotomal recording sites, prompting a surgical and anesthesia alert for evolving positional injury to the left brachial plexus as depicted in Figure 3. There was no such amplitude loss for the non-operative right side tceMEP responses. While not as dramatic as the left tceMEP amplitude attenuation, the left ulnar nerve cortical somatosensory evoked potential amplitude also decreased by $50 \%$ relative to baseline (Figure 3 ). Here again, the contralateral control remained unchanged.

The left arm was repositioned immediately several times in an effort to reduce brachial plexus stretch/compression. Mean arterial pressure also was elevated from 75 to $96 \mathrm{~mm} \mathrm{Hg}$ in an effort to optimized peripheral perfusion. Despite repeated attempts at adjusting arm position from 180 degrees to 90 degrees with the robotic guidance system in place, tceMEPs from the left upper extremity remained highly attenuated across all mytomal recording sites on the left with no such changes on the right upper extremity.

As a result of the clear neurophysiologic evidence of evolving left brachial plexopathy with the arm abducted even at 90 degrees, the decision was made to remove the robotic guidance system to allow for placement of the left arm in a neutral 
position by the patient's side. At this time, the surgical strategy was changed and the thyroid was exposed via a direct open approach to complete resection of the left lobe.

Approximately 50 minutes thereafter, left tceMEP and ulnar nerve SSEP amplitudes began to show remarkable recovery with almost complete resolution by the end of closing (Figure 4). Post-operatively the patient had full range of motion, normal sensation and $5 / 5$ upper extremity muscle strength on neurologic examination.

\section{Discussion}

Intraoperative positioning to prevent neuropraxia is a fundamental principle that has been recognized for centuries. Understanding the mechanisms of injury and techniques to limit such morbidities is a prerequisite for safe care of the surgical patient. The development of new surgical techniques often results in an increase in complexity with the potential for unforeseen harm. We have demonstrated how intraoperative neuromonitoring during transaxillary thyroid surgery can identify a developing brachial plexopathy and enable changes in technique to avoid a potentially debilitating morbidity.

Brachial plexus injury in adults may involve hyperextension of the neck with the arm fully abducted and anteriorly extended beyond the frontal plane, as seen in the transaxillary thryoidectomy operative position. One explanation for this injury with the arm fully abducted is the impedance of venous drainage causing swelling of the surrounding tissue and severe compression of the brachial plexus against the clavicle and anterior scalene muscle ${ }^{\text {viii. }}$. We suggest that patients with a high BMI and a relatively short neck, such as the one described herein, may be predisposed to such injuries. 
Often the isolation of poorly functioning specific muscle groups supplied by the brachial plexus leads the clinician to isolate the rootlets involved. In the present example the attenuation of tceMEPs from the left deltoid, biceps, extensor carpi radialis, triceps and first dorsal interosseous muscles indicated that the entirety of the plexus was under distress. Function did not return immediately when the arm's neutral position was reestablished, but recovery of both sensory and motor attenuation was seen approximately 50 minutes later, following transition to an open procedure. Comparison to the unaffected evoked potential amplitudes in the contralateral arm as well as to the normal baseline responses validated that the upper extremity amplitude loss on the operative side represented a harbinger of positional brachial plexopathy if left unattended. Tolerance of peripheral nerves to stretch and/or compression depends on both the magnitude and duration of mechanical distortion. Stretch that exceeds $10 \%$ to $20 \%$ may result in structural damage; moreover, prolonged stretch of sufficient magnitude can result in ischemic damage (Grant et al. 1999). Prompt reversal of excessive stretch/compression can lead to recovery of function. Lyon et al. (2009) demonstrated that 10 minutes of lumbar spinal nerve root retraction at 2 Newtons produced an average 59\% reduction in tceMEP amplitude from baseline. Ten minutes after release of retraction, response amplitudes increased to within $20 \%$ of baseline on average. The results of the present study emphasize that while the precise etiology of neurophysiological signal change (i.e. mechanical vs. ischemic) may be difficult to establish, and time course of recovery difficult to predict in specific cases, prompt identification and reversal of the proximate cause of signal change can result in 
recovery of neurophysiologic function and avoidance of post-operative neurologic sequelae.

Given the low incidence of complications accompanying conventional and minimally invasive thyroid surgery, proponents of transaxillary robotic thyroidectomy must demonstrate a similar commitment to patient safety. During a preoperative checklist involving our nursing and anesthesia teams, we identified the potential risks of positioning and posited the need for neuromonitoring of the brachial plexus. Such monitoring resulted in avoidance of a potentially significant brachial plexopathy. Unless similarly effective countermeasures can be established or the incidence of brachial plexopathy or other peripheral neuropathy during transaxillary thyroidectomy can be shown to approach less than $0.1 \%$, we recommend that brachial plexus monitoring be adopted as the standard of care in transaxillary robotic thyroidectomy. If the risk to the brachial plexus is to be reduced by neuromonitoring, it raises the question whether this additional cost to the robotic surgery counterbalances the benefits of this technology.

\section{Conclusion:}

We present the first case of multimodality intraoperative neurophysiologic monitoring detection of evolving brachial plexopathy during transaxillary robotic thyroidectomy. Rapid identification of this potentially debilitating complication served to facilitate immediate rescue intervention by returning the affected arm to a neutral position and converting the procedure to an open thyroidectomy.. Given the potential for brachial plexus injury we recommend that continuous tceMEP and SSEP monitoring be considered during such procedures. 

Acknowledgement: Special thanks to Annette Jablonski, Carol Shindle and Ankit Pahwa for their contributions to manuscript.

\section{Figure Legend:}

Figure 1: Patient positioned with arm in full abduction.

Figure 2: Baseline transcranial electric motor evoked potentials from left and right deltoid, biceps, extensor carpi radialis, triceps and first dorsal interosseous muscles, and cortical somatosensory evoked potentials elicited to left and right ulnar nerve stimulation.

Figure 3: Loss of left upper extremity tceMEP amplitudes across all myotomal recording sites (red arrow suggesting evolving positional left brachial plexopathy. Note that the left Ulnar nerve somatosensory evoked potential amplitudes also decreased by $\geq 50 \%$ relative to baseline.

Figure 4: Resolving left tceMEP and ulnar nerve SSEP amplitudes approximately 50 minutes after placing the left arm in the neutral position for conversion to an open procedure. 
References

1) Practice advisory for the prevention of perioperative peripheral neuropathies: American Society of Anesthesiologists Task Force on Prevention of Perioperative Peripheral Neuropathies: Anesthesiology 2000; 92: 1168-1182.

2) Practice Advisory for the Prevention of Perioperative Peripheral Neuropathies: An updated report by the American Society of Anesthesiologists. Task Force on Prevention of Perioperative Peripheral Neuropathies. Anesthesiology 2011; 114: 1-14.

3) Schwartz DM, Drummond DS, Hahn M, Ecker ML, Dormans JP. Prevention of positional brachial plexopathy during surgical correction of scoliosis. J Spinal Disord. 2000; 13: 178-82.

4) Schwartz DM, Sestokas AK, Hilibrand AS, et al. Neurophysiological identification of position-induced neurologic injury during anterior cervical spine surgery. J Clin Monit Comput. 2006; 20: 437-44.

5) Bhalodia VM, Sestokas AK, Tomak PR, Schwartz DM. Transcranial electric motor evoked potential detection of compressional peroneal nerve injury in the lateral decubitus position. J Clin Monit Comput 2008; 22:319-26.

6) Hickey C, Gugino LD, Aglio LS, Mark JB, Son SL, Maddi R. Intraoperative somatosensory evoked potential monitoring predicts peripheral nerve injury during cardiac surgery. Anesthesiology 1993; 78:29-35. 
7) Kang SW, Lee SC, Lee SH et al. Robotic thyroid surgery using a gasless transaxillary approach and the da Vinci S system: The operative outcomes of 338 consecutive patients. Surgery $2009 ; 146: 1048-55$.

8) Zaneteas, PD. Brachial Plexus injuries and the electrodiagnostic examination. Current Sports Medicine 2003; $2: 7-14$

9) Grant GA, Goodkin R, Kliot M. Evaluation and surgical management of peripheral nerve problems. Neurosurgery. 1999;44(4):825-39; discussion 839-40.

10) Lyon R, Lieberman JA, Feiner J, Burch S. Relative efficacy of transcranial motor evoked potentials, mechanically-elicited electromyography, and evoked EMG to assess nerve root function during sustained retraction in a porcine model. Spine (Phila Pa 1976). 2009;34(16):E558-64. 